GA-C20806

\title{
TECHNICAL PROGRESS REPORT AN EDGE DENSITY FLUCTUATION DIAGNOSTIC FOR DIII-D USING LITHIUM BEAMS
}

\author{
by \\ DAN M. THOMAS
}

Prepared under

Grant No. DE-FG03-90ER54081

for the U.S. Department of Energy

DECEMBER 1991

\section{GENERAL ATOMICS}




\section{DISCLAIMER}

This report was prepared as an account of work sponsored by an agency of the United States Government. Neither the United Staies Government nor any agency thereof, nor any of their employees, makes any warranty, express or implied, or assumes any legal liability or responsibility for the accuracy, sompleteness, or usefulness of any information, apparatus, product, or process disclosed, or represents that its use would not infringe privately owned rights. Reference herein to any specific commercial product, process, or service by trade name, trademark, manufacturer, or otherwise, does not necessarily constitute or imply its endorsement, recommendation, or favoring by the United States Government or any egency thereof. The views and opinions of authors expressed herein do not necessarily state or reflect those of the United States Government or any agency thereof. 


\title{
TECHNICAL PROGRESS REPORT AN EDGE DENSITY FLUCTUATION DIAGNOSTIC FOR DIII-D USING LITHIUM BEAMS
}

\author{
by \\ DAN M. THOMAS
}

Prepared under

Grant No. DE-FG03-90ER54081

for the U.S. Department of Energy

\author{
GENERAL ATOMICS PROJECT 3879 \\ DECEMBER 1991
}

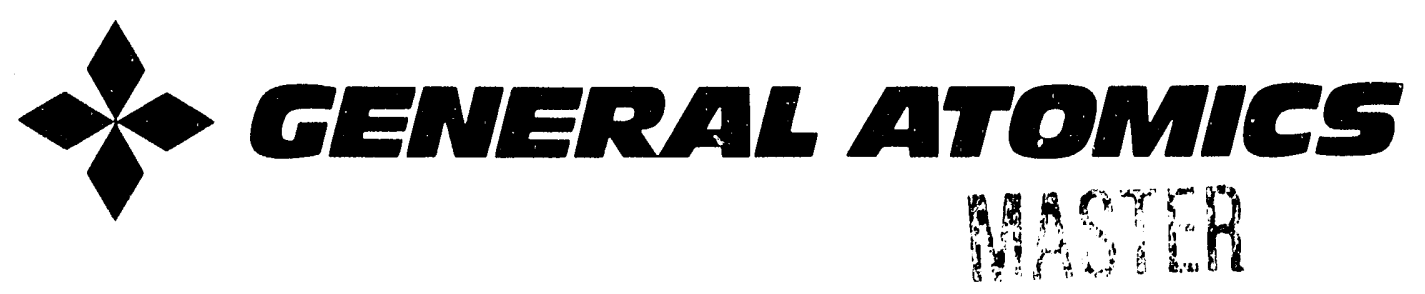




\section{INTRODUCTION}

This report covers the research conducted under DOE grant DE-FG03-90ER54081 during the period August 15, 1990 through November 15, 1991. Progress during the period March 15, 1990 through August 15, 1990 was covered in a previous report. Highlights during this period include the development of a compact neutral lithium accelerator capable of producing several $\mathrm{mA}$ at up to $30 \mathrm{kV}$, measurements of intrinsic beam fluctuation levels, and the design and partial completion of the diagnostic installation on the DIII-D tokamak. We also had one journal article describing the system published in Reviews of Scientific Instruments, presented a poster on our recent progress at the APS Plasma Physics conference, and submitted an abstract to the 9th Topical Conference on Plasma Diagnostics.

The overall objective of this project is to provide detailed information about the behavior of the electron density in the edge region of DIII-D, and in particular to examine the local character of the associated degradation in confinement properties. Measurements should provide important data for testing theories of the L-H transition in tokamaks and should help in assessing the role of various instabilities in anomalous transport. The work on this project may be naturally organized according to the following six subareas: Ion source/beam system, neutralizer system, optical system, data acquisition, data analysis, and machine (DIII-D) interface. Progress in each of these areas will be discussed briefly. We also briefly discuss our plans for future work on this program. 


\section{ION SOURCE AND BEAM DEVELOPMENT}

The ion source is necessary to provide a dense, monoenergetic, focussed beam of lithium ions for subsequent neutralization and injection into the tokamak periphery. During the current period, we built up a lithium beam laboratory with a test stand on which we operated several different beamline geometries for extraction and formation of intense low energy lithium ion beams. The laboratory also includes a vacuum coating stand for manufacturing our large area $(5 \mathrm{~cm}$ diameter) thermoemissive lithium ion sources. We have constructed a compact ion accelerator which is simple to operate. The ion optics are similar to those chosen for the previously built ASDEX lithium beam upgrade, but we added an electrostatic einzel lens downstream from the initial extraction region to refocus the beam. This permits a degree of control over the beamspread due to space charge expansion and significantly increases the current transmitted to the neutralizer region. Ion currents of greater than $10 \mathrm{~mA}$ have been focused and transmitted to a faraday cup placed downstream from the neutralizer region. 


\section{NEUTRALIZER}

The neutralization of $\mathrm{Li}^{+}$ions to $\mathrm{Li}$ neutrals must be done quickly enough (i.e., over a short enough path length) such that the ion beam does not get a chance to expand too much. We have developed techniques for efficiently neutralizing the ions using charge transfer in sodium vapor, and charge transfer cells utilizing recycling of the condensing sodium vapor for extended lifetime have been constructed and tested. The neutralizer cell provides a localized region of sodium vapor where the incident lithium ions may efficiently charge exchange and form neutrals with minimaı scattering. A temperature controlled heater block surrounds the central section of the tube and serves a dual purpose. First it provides precise control of the sodium vapor pressure and hence neutralization efficiencies. Second, by maintaining the proper temperature gradient between the hot center and the cool ends, and lining the inside of the tube with a nickel mesh wick, the sodium vapor effuses from the central section through capillarity, analogous to a heat pipe. This effect minimizes the loss of sodium out through the ends of the cell, and considerably extends the operational lifetime. Using this type of cell and a detector sensitive to both ions and neutrals, neutralization efficiencies of greater than $80-90 \%$ have been demonstrated. Our efforts have resulted in the capability of producing stable neutral beam pulses of several seconds' duration and several mA-equivalent neutral current. 


\section{OPTICAL SYSTEM}

The two methods currently envisioned for obtaining the fluorescence data are a multichannel system based on discrete detectors, and an imaging system based on a gated, intensified CCD camera. For the discrete multichannel system, detection of the lithium fluorescence with sufficient bandwidth to accurately analyze the plasma-induced fluctuations requires both sensitive, low noise detectors in the visible region of the spectrum and a quiet neutral beam (low intrinsic fluctuations). We have chosen Si photodiodes as the detector element due to their high quantum efficiency $(85 \%$ at $670.8 \mathrm{~nm})$ and low noise. Tests have been conducted using $0.8 \mathrm{~mm}$ diameter diodes and a preamplifier board supplied by the Phaedrus group at $\mathrm{U}$. Wisconsin, Madison. Measurements of the intrinsic beam noise were made on the test stand using background neutral gas collisions to provide a small fluorescence signal. A comparison of noise levels before and after beam turnoff indicates detector noise totally dominates any beam noise at these signal levels. Note that, due to the much higher collision rate which obtains for electrons, we would expect substantially higher signals from an actual plasma injection (for a similar intensity beam). We are constructing three similar detector channels for initial data-taking on the tokamak. 


\section{DATA ACQUISITION}

A microVAX/CAMAC-based high speed data acquisition system capable of processing 16 channels of $1 \mathrm{MHz}$-bandwidth data has been assembled and tested in the laboratory, and has been used in characterizing the intrinsic beam fluctuations and detector performance (see átuve). This computer will also serve as the local control for beam operation and beamline vacuum interlocks. The system has been integrated into the DIII-D data acquisition VAX cluster. 


\section{DATA ANALYSIS}

In order to understand the relationship between the emitted light and the underlying density profile we have begun by evolving an eight-state collisional-radiative beam-plasma model. A computer code based on this model is capable of accurately predicting the beam penetration and fluorescence emission profile for lithium beam energies between $5 \mathrm{keV}$ and a few $\mathrm{MeV}$, given input density and temperature profiles. The code has been calibrated with experimental light profiles obtained from the TEXT lithium beam which we modified to yield a $15-30 \mathrm{keV}$ beam energy. Very good agreement was found between the code prediction and the observed fluorescence profiles for plasma densities up to $7 \times 10^{13} \mathrm{~cm}^{-3}$. A preliminary unfolding algorithm based on only two levels has been identified and tested against mock profiles generated by the beam penetration code using artificial density fluctuations. This method shows promise as it is able to reproduce the input density profile to within a few percent. A full test of the method awaits the arrival of actual data. 


\section{MACHINE INTERFACE}

During the last few months a considerable amount of work has been accomplished on the tokamak interface and the necessary parts for installing the beamline and viewing port to take advantage of the vent period this winter on DIII-D. The injection port at $75 R 0$ was modified for an 8 " gate valve and a small auxiliary window was installed to permit observation of the beam by a CCD camera. We took this opportunity to replace the existing protective tiles on the inside of this port with graphite tiles. This port modification is fully compatible with the future upgrade to a 10-cm high strip beam. The corresponding observation port at $90 \mathrm{R} 0$ has also been modified to provide a shuttered window with a sacrificial sapphire plate for additional protection. This choice of ports provides maximum efficiency of light collection without extensive modification of the vessel wall itself. The port arrangement offers extremely good tangential view of the edge region and should provide an ideal environment for imaging in both the poloidal and radial directions. We will be able to use a $7.5 \mathrm{~cm}$-diameter lens positioned approximately $85 \mathrm{~cm}$ from the predicted beam position. All the vacuum interface components have been installed with the exception of the two graphite tiles, which will be installed after the initial alignment of the external viewing optics. A new personnel deck was installed in the region around 60R0-75R0, and the front and rear beamline support stands have also been installed and roughly aligned. In order to eliminate deflection of the low energy ion beam before it is neutralized, a magnetic shield box has been designed and is undergoing the final heat treatment before being assembled on the rear stand. In the near future we hope to install the entire beamline in the pit and perform an initial pumpdown and check of the vacuum control system.

Twenty-one $0.8 \mathrm{~mm}$-diameter optical fibers have been installed to carry the fluorescence light from the collection optics to individual filter/detector modules located in the GRECE room outside of the DIII-D nedtron shield wall. The fibers are mounted so as to image 17 radial and 4 poloidal positions. The collection area for each fiber is a $6 \mathrm{~mm}$ diameter circle separated from each of its neighbors by $7 \mathrm{~mm}$. The total extent of the radial array is $110 \mathrm{~mm}$ and has been aligned during the vent to cover the region $\left(r_{\text {sep }}+20 \mathrm{~mm}\right)$ to $\left(r_{\text {sep }}-90 \mathrm{~mm}\right)$. 


\section{PROGRAM PLAN FOR BY-3}

The program plans for the grant extension period are essentially the same as those outlined in the original proposal, with no change in the statement of work and no major shifts of emphasis planned. As mentioned previously, we are in the middle of installing the diagnostic on the tokamak and should be able to begin taking data in the spring of 1992 .

Our plans for future work include completing the installation and shakedown of the diagnostic once it is installed. Once the system is operating reliably we will commence acquisition of high speed multichannel data using three detector channels under a variety of tokamak discharge conditions. The initial emphasis will be on the measurement of radial correlation lengths for $\mathrm{L}$ and $\mathrm{H}$ mode discharges. Data analysis and handling codes remain to be written and further modeling efforts will be made. Based on detector performance and the actual signal levels, the full complement of 16 detector channels will be constructed and installed. Once the detectors are well characterized, a simple modification of the optical filter should permit a proof-of-principle experiment to be done measuring carbon and/or hydrogen densities in the edge. Indeed, this measurement is to be the major emphasis of the ASDEX-U lithium beam program at Garching. A logical first step towards imaging of coherent structures in the edge is to take a snapshor of the 1-D beam using a gated, intensified camera with a GaAs photocathode. The beam may be defocussed to provide a somewhat larger poloidal extent if need be. We plan to continue ion source testing in the laboratory; in particular, tests of ion extraction and beam formation from our largest $\left(5 \times 10 \mathrm{~cm}^{2}\right)$ source plates as the first step towards a $1 \times 10 \mathrm{~cm}$ strip neutral beam for subsequent 2-D studies. 

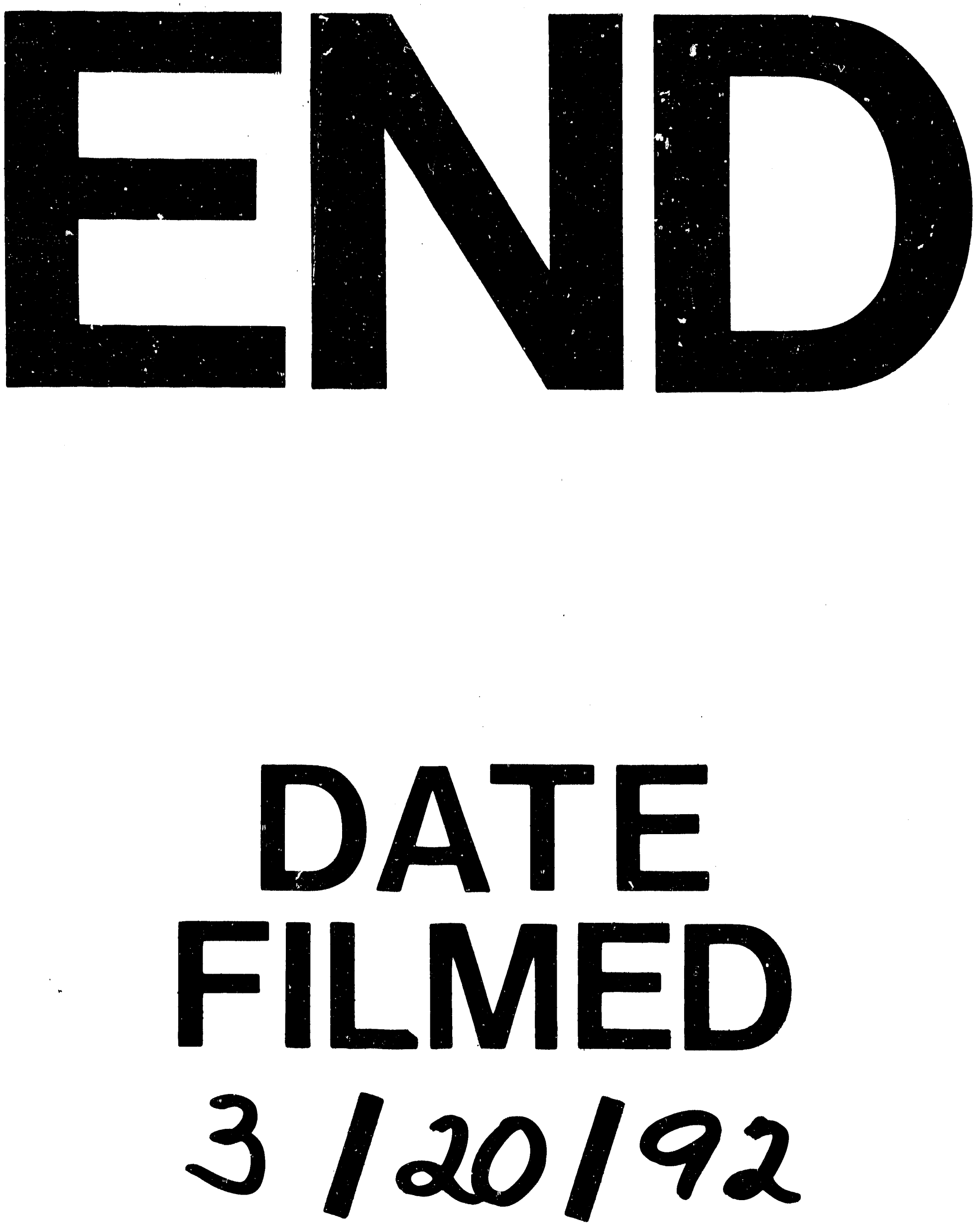